\title{
FAITHFUL IRREDUCIBLE REPRESENTATIONS OF MODULAR LIE ALGEBRAS
}

\author{
DONALD W. BARNES
}

\begin{abstract}
Let $L$ be a finite-dimensional Lie algebra over a field of characteristic $p \neq 0$. By a theorem of Jacobson, $L$ has a finite-dimensional faithful completely reducible module. We show that if $F$ is not algebraically closed, then $L$ has an irreducible such module. We also give a necessary and sufficient condition for a finite-dimensional Lie algebra over an algebraically closed field of non-zero characteristic to have a faithful irreducible module.
\end{abstract}

\section{INTRODUCTION}

Let $L$ be a finite-dimensional Lie algebra over the field $F$ of characteristic $p>0$. By Jacobson's Theorem, [6, Theorem VI.2], [8, Theorem 5.5.2], $L$ has a finitedimensional faithful completely reducible module. We adapt Jacobson's proof to show that if $F$ is not algebraically closed, then $L$ has an irreducible such module. We may replace $L$ with a minimal $p$-envelope. Thus without loss of generality, we may assume that $L$ is restricted. Further, by [3, Corollary 2.3], we may assume that the $p$-operation $[p]$ vanishes on $\operatorname{Asoc}(L)$, the abelian socle of $L$. We note that, by the Artin-Schreier Theorem[1, (see [7, Theorem 11.14] or [4, Theorem 3.1],) our assumption that $F$ is not algebraically closed implies that the algebraic closure $\bar{F}$ has infinite dimension over $F$.

Jacobson takes an appropriately selected character $c$ and uses the $c$-reduced enveloping algebra $\mathrm{u}(L, c)$ to obtain for a given element $x \in L$, an irreducible module on which $x$ acts non-trivially. If $x$ is in some minimal ideal $K$ of $L$, then $K$ is not in the kernel of the representation. For each minimal ideal $K$, we can clearly construct an irreducible module $V_{K}$ on which $K$ acts non-trivially. We shall choose modules $V_{K}$ such that their tensor product has a composition factor on which all minimal ideals act non-trivially. It is in the making of this choice for the abelian minimal ideals that we use that the dimension of $\bar{F}$ over $F$ is infinite.

\section{DIAGONALS}

Let $A_{1}, \ldots, A_{r}$ be abelian minimal ideals of $L$ which, as $L$-modules, are isomorphic with isomorphisms $\phi_{i}: A_{1} \rightarrow A_{i}, \phi_{1}=$ id. For a given $A_{1}$, we choose the $A_{i}$ with $r$ as large as possible subject to their sum being direct. With the $A_{i}$ so chosen, we say that $A_{1}$ has multiplicity $r$ in $\operatorname{Asoc}(L)$. For any $\lambda_{1}, \ldots, \lambda_{r}$, not all zero, the set $\left\{\sum_{i=1}^{r} \lambda_{i} \phi_{i}(a) \mid a \in A_{1}\right\}$ is also a minimal ideal and every minimal

2010 Mathematics Subject Classification. Primary 17B50.

Key words and phrases. modular Lie algebras, faithful representations.

This work was done while the author was an Honorary Associate of the School of Mathematics and Statistics, University of Sydney.

${ }^{1}$ I wish to thank J. M. Bois for drawing my attention to this theorem. 
ideal isomorphic to $A_{1}$ has this form. Those with more that one of the $\lambda_{i}$ non-zero are called diagonals. We shall denote the ideal given by $\lambda=\left(\lambda_{1}, \ldots, \lambda_{r}\right)$ by $A_{\lambda}$.

Under our assumptions, an abelian minimal ideal $A$ acts non-trivially on an irreducible module $V$ with character $c$ if and only if $c(A) \neq 0$. If for the above $A_{i}$, we put $c_{i}=c \mid A_{i}$, then for $a_{\lambda}=\sum \lambda_{i} \phi_{i}(a) \in A_{\lambda}$, we have $c\left(a_{\lambda}\right)=\sum \lambda_{i} c_{i}\left(\phi_{i}(a)\right)$. Thus $c$ vanishes on some $A_{\lambda}$ if and only if the $c_{i} \phi_{i}$ are linearly dependent.

We shall use generalised characters, linear maps $c: L \rightarrow \bar{F}$, where $\bar{F}$ denotes the algebraic closure of $F$. If $c$ is a character of a module $V$ then every conjugate of $c$ is also a character. The set of all characters of composition factors of $\bar{V}=\bar{F} \otimes V$ is called the character cluster $\mathrm{Cl}(V)$. (See [1.) If the restriction $\mathrm{Cl}(V) \mid A$ of $\mathrm{Cl}(V)$ to the abelian minimal ideal $A$ does not contain 0, then $A$ acts non-trivially on every composition factor of $V$. By [1, Theorem 3.4], if $V$ is irreducible, then $\mathrm{Cl}(V)$ is simple, that is, the characters in $\mathrm{Cl}(V)$ are all conjugate. Thus if $c$ is a generalised character of the irreducible module $V$ and $c(A) \neq 0$, then $A$ acts non-trivially on $V$.

If $C$ is a simple cluster, then we can form the $C$-reduced enveloping algebra $\mathrm{u}(L, C)$. For any $x \in L$, the element $x^{p}-x^{[p]}$ of the universal enveloping algebra is central and on an $\bar{F} \otimes L$-module with character $c$, acts as multiplication by $c(x)^{p}$. Let $m_{x}(t) \in F[t]$ be the minimal polynomial of $c(x)^{p}$. The $C$-reduced enveloping algebra $\mathrm{u}(L, C)$ is the quotient of $\mathrm{U}(L)$ by the ideal generated by $\left\{m_{x}\left(x^{p}-x^{[p]}\right) \mid\right.$ $x \in L\}$ for some $c \in C$. (Since all $c \in C$ are conjugate, they all give the same polynomial $m_{x}(t)$.) The $C$-reduced enveloping algebra is finite-dimensional. Any $\mathrm{u}(L, C)$-module is an $L$-module with cluster $C$. This construction may be found in Farnsteiner [5] or in Barnes [2].

Lemma 2.1. For any simple cluster $C$, there exists a finite-dimensional irreducible $L$-module with cluster $C$.

Proof. As $\mathrm{u}(L, C)$ is a $\mathrm{u}(L, C)$-module, any $L$-module composition factor of $\mathrm{u}(L, C)$ is an irreducible module with cluster $C$.

Lemma 2.2. There exists an irreducible $L$-module $V_{0}$ on which every abelian minimal ideal acts non-trivially.

Proof. Let $A_{1}, \ldots, A_{r}$ be abelian minimal ideals of $L$ which are isomorphic with isomorphisms $\phi_{i}: A_{1} \rightarrow A_{i}, \phi_{1}=\mathrm{id}$, chosen as above. As $\operatorname{dim}(\bar{F} / F)$ is infinite, we can take $r$ linearly independent linear maps $f_{i}: A_{1} \rightarrow \bar{F}$ and by setting $g_{i}=$ $f_{i} \circ \phi_{i}^{-1}: A_{i} \rightarrow \bar{F}$, construct a map $g: \sum_{i} A_{i} \rightarrow \bar{F}$ which is non-zero on every minimal ideal isomorphic to $A_{1}$. Doing this for each isomorphism type of abelian minimal ideal, we obtain $c: \operatorname{Asoc}(L) \rightarrow \bar{F}$ which is nonzero on every abelian minimal ideal. This map can be extended to a generalised character and so we obtain an irreducible module $V_{0}$ on which every abelian minimal ideal acts nontrivially.

\section{The MAIn RESUlT}

Lemma 3.1. Let $K$ be an ideal of $L$ and let $V, W$ be irreducible $L$-modules. Suppose that $K$ acts non-trivially on $V$ and trivially on $W$. Then $K$ acts non-trivially on every composition factor of $V \otimes W$.

Proof. Regarded as $K$-module, $V \otimes W$ is a direct sum of copies of $V$. Thus any composition factor of $V \otimes W$ is as $K$-module, a direct sum of copies of $V$. 
Theorem 3.2. Let $L$ be a finite-dimensional Lie algebra over a field $F$ of characteristic $p \neq 0$. Suppose that $F$ is not algebraically closed. Then there exists a finite-dimensional faithful irreducible L-module.

Proof. Let $K_{1}, \ldots, K_{s}$ be the non-abelian minimal ideals of $L$. Then $K_{i}$ is an irreducible $L$-module on which $K_{i}$ acts non-trivially and on which every other minimal ideal acts trivially. We start with $V_{0}$ given by Lemma 2.2 and construct inductively irreducible modules $V_{i}$ on which the abelian minimal ideals and the $K_{j}$ for $j \leq i$ all act non-trivially.

We suppose that we have constructed $V_{i-1}$. If $K_{i}$ acts non-trivially on $V_{i-1}$, we set $V_{i}=V_{i-1}$. If not, then we take for $V_{i}$ any composition factor of $V_{i-1} \otimes K_{i}$. By Lemma 3.1, this satisfies the requirements.

Put $V=V_{s}$. Then $V$ is a finite-dimensional irreducible $L$-module on which every minimal ideal of $L$ acts non-trivially. Since the kernel of the representation cannot contain any minimal ideal, the representation is faithful.

When the field $F$ is algebraically closed field, the above argument leads to a necessary and sufficient condition for the existence of a faithful irreducible module.

Theorem 3.3. Let $L$ be a finite-dimensional Lie algebra over the algebraically closed field $F$ of characteristic $p \neq 0$. Then $L$ has a faithful irreducible module if and only if, for each abelian minimal ideal $A$ of $L$, the L-module isomorphism type of $A$ has multiplicity $r \leq \operatorname{dim}(A)$.

Proof. We show first that if $L$ satisfies the condition, then so does a minimal $p$ envelope $\left(L^{e},[p]\right)$ of $L$. We may suppose that $[p]$ vanishes on $\operatorname{Asoc}(L)$. Suppose that $A$ is an abelian minimal ideal of $L^{e}$. Then $A$ is a $[p]$-ideal and $L^{e} / A$ is again a restricted Lie algebra. If $A \nsubseteq L$, then $L^{e} / A$ is a $p$-envelope of $L$ of smaller dimension. Thus every minimal ideal of $L^{e}$ is contained in $L$ and again, without loss of generality, we may suppose that $L$ is restricted with $[p]$ vanishing on $\operatorname{Asoc}(L)$.

We have already shown that the condition is sufficient for the existence of a faithful irreducible $L$-module. So suppose that $V$ is a faithful irreducible $L$-module. By [8, Theorem 5.2.5], $V$ has a character $c$. Let $A_{1}, \ldots, A_{r}$ be abelian minimal ideals chosen as above, with $L$-module isomorphisms $\phi_{i}: A_{1} \rightarrow A_{i}$. We have the linear maps $c_{i}=c \circ \phi_{i}: A_{1} \rightarrow F$. For the diagonal $A_{\lambda}, c \mid A_{\lambda}=\sum \lambda_{i} c_{i} \circ \phi^{-1}$. As $V$ is faithful, $c$ is non-zero on every $A_{\lambda}$, so the $c_{i}$ are linearly independent. Therefore $r \leq \operatorname{dim}\left(A_{1}\right)$.

Examples of algebras not satisfying the condition are easily found. The 2dimensional abelian algebra is one such.

Corollary 3.4. Let $L$ be a finite-dimensional Lie algebra over an algebraically closed field of characteristic $p \neq 0$. Then $L$ is a quotient of an algebra which has a faithful irreducible module.

Proof. $L$ has a faithful completely reducible module $V$. We may suppose that $V$ has no redundant summands. The split extension of $V$ by $L$ satisfies the condition for the existence of a faithful irreducible module.

\section{REFERENCES}

1. D. W. Barnes, Character clusters for Lie algebra modules over a field of non-zero characteristic, Bull. Aust. Math. Soc. 89 (2014), 234-242. Also arXiv:1301.4710 
2. D. W. Barnes, Induced modules for modular Lie algebras, arXiv:1312.0163 55, (2016).

3. D. W. Barnes, Faithful completely reducible representations of modular Lie algebras, Comm. Algebra 45 (2017), 2629-2632. Also arXiv:1603.01894

4. K. Conrad, www.math.uconn.edu/ kconrad/blurbs/galoistheory/artinschreier.pdf

5. R. Farnsteiner, Extension functors of modular Lie algebras, Math. Ann. 288 (1990), 713-730.

6. N. Jacobson, Lie algebras Interscience, New York-London (1962).

7. N. Jacobson, Basic Algebra II, 2nd ed., W. H. Freeman and Co., New York, 1989.

8. H. Strade and R. Farnsteiner, Modular Lie algebras and their representations, Marcel Dekker, Inc., New York-Basel, 1988

1 Little Wonga Rd., Cremorne NSW 2090, Australia,

E-mail address: D.Barnes@maths.usyd.edu.au 